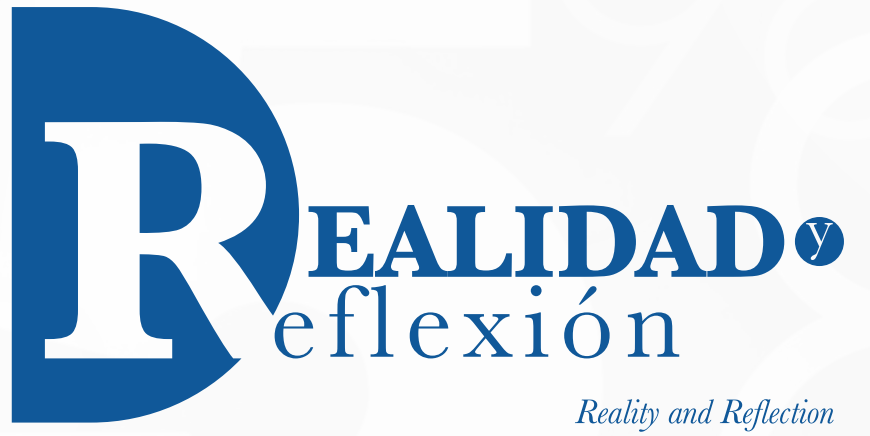

ISSN 1992-6510

Año 21, N 53, San Salvador, El Salvador, Centroamérica. Revista Semestral Enero-Junio 2021

YEAR 21, N53, SAN SALVADOR, EL SALVADOR, CENTRAL AMERICA. SEMESTRAL JOURNAL JANUARY-JUNE 2021

\title{
Los niños que calculaban: en situación de riesgo social y de exclusión del aprendizaje matemático ${ }^{1}$
}

As crianças que calculavam: em situação de risco social e de exclusão de aprendizagem matemática

Children who counted: in social risk situations and of mathematics learning exclusion

Cristiano Alberto Muniz Facultad de Educación Universidad de Brasilia, Brasil. cristianoamuniz@gmail.com

Recibido: 29 de junio de 2020 Aceptado: 1 de septiembre de 2020 DOI: $10.5377 /$ ryr.v53i53.10888

(๐) $\otimes \odot$

$1 \mathrm{El}$ título original es "AS CRIANÇAS QUE CALCULAVAM: em situação de risco social e de exclusão de aprendizagem matemática" del autor Cristiano Muniz. Texto traducido del portugués al español por Jeser Candray en octubre de 2020 con autorización del autor para este número especial. 


\section{Resumen}

La búsqueda por la comprensión de la constitución del ser matemático como sujeto que aprende y produce sentidos subjetivos de su capacidad en elaborar conocimientos matemáticos encuentra en la categoría "sujeto que aprende", de la Teoría de la Subjetividad - TS, un concepto que amplia como profundiza los análisis interpretativos de la constitución matemática de los niños. La TS posibilita la ampliación de la comprensión del fenómeno del aprendizaje matemático más allá del análisis estrictamente cognitivo, de análisis microgenética de las producciones de registros matemáticos, lo que realizamos en las últimas décadas apoyados en la Teoría de los Campos Conceptuales. Apoyados en diálogos producidos en talleres de matemática lúdica y en la conversación con madres sobre sus historias educativas, buscamos sentidos subjetivos en la historia del aprendizaje matemático de cuatro niños en situación de riesgo. Además del diálogo establecido a lo largo de los talleres lúdicos, recolectamos sus producciones matemáticas. Ese registro, sumado a los constantes diálogos en contexto, permitieron el análisis y la explicación de esquemas matemáticos implícitos en esas producciones matemáticas y reflejaron importantes elementos constituidores de los complejos procesos de aprendizaje matemático. Ahora bien, los análisis microgenéticos no fueron suficientes para el develar la compleja dimensión simbólica-emocional de significación de aprendizajes matemáticos por los sujetos. El diálogo de los niños y sus respetivas madres, por medio de conversación videograbada, permitió comprender mejor la naturaleza de las producciones y sus significados más allá de la situación de la actividad lúdica. Este artículo se limita a traer al análisis de uno de esos casos investigados, un niño con deficiencia auditiva y que, por tanto, presenta dificultad en la organización de los algoritmos matemáticos.

Palabras clave: niños calculan, configuraciones subjetivas, aprendizaje matemático, aprendizaje e inclusión.

\section{RESUMO}

A busca pela compreensão da constituição do ser matemático como sujeito que aprende e produz sentidos subjetivos de sua capacidade em elaborar conbecimentos matemáticos encontra na categoria "sujeito que aprende", da Teoria da Subjetividade-TS, um conceito que tanto amplia quanto aprofunda as análises interpretativas da constituição matemática de crianças. A TS possibilita ampliação da compreensão do fenômeno da aprendizagem matemática para além da análise estritamente cognitiva, de análise microgenética das produçóes de registros matemáticos, o que realizamos nas últimas décadas apoiados na Teoria dos Campos Conceituais. Apoiados em diálogos produzidos em oficinas matemáticas lúdicas e na conversação com mães sobre suas histórias educativas, buscamos sentidos subjetivos na história de aprendizagem matemática de quatro crianças em situação de risco. Além do diálogo estabelecido ao longo das oficinas lúdicas, coletamos suas produções matemáticas. Esses registros, somados aos constantes diálogos em contexto, permitiram a análise e a explicitação de esquemas matemáticos implicitos nessas produções matemáticas e revelaram importantes elementos constituidores dos complexos processos de aprendizagem matemática. Entretanto, as análises microgenéticas não foram suficientes para o desvelamento da complexa dimensão simbólico-emocional de 
significação de aprendizagens matemáticas pelos sujeitos. $O$ diálogo com as crianças e suas respectivas mães, por meio de conversão videogravada, permitiu melhor compreender a natureza das produçôes e seus significados para além da situação da atividade lúdica. Este artigo se limita a trazer a análise de apenas um desses casos investigados, de um menino com deficiência auditiva e que, portanto, apresenta dificuldade na organização dos algoritmos matemáticos.

Palavras-chave: crianças calculam; configurações subjetivas; aprendizagem matemática; aprendizagem e inclusão;

\section{ABSTRACT}

The search for understanding the constitution of the mathematical being as a subject who learns and produces subjective meanings of their ability to develop mathematical knowledge finds, in the category "subject who learns", of the Theory of Subjectivity-TS, a concept that both broadens and deepens the interpretive analyses of the mathematical constitution of children. The TS allows to expand the understanding of the phenomenon of mathematical learning beyond the strictly cognitive analysis, of microgenetic analysis of the mathematical records production, which we have carried out in the last decades supported by the Theory of Conceptual Fields. Supported by conversations that we obtained in playful mathematical workshops and with mothers about their educational histories, we sought subjective meanings in the history of the mathematical learning of four children at risk. In addition to the dialogue established during the playful workshops, we collected their mathematical productions. These records, added to the constant dialogues in context, allowed the analysis and explanation of mathematical schemes implicit in these mathematical productions and revealed important elements of complex mathematical learning processes. However, the microgenetic analyses were not enough to unveil the complex symbolic-emotional dimension of mathematical learning meaning by the subjects. The dialogue with the children and their mothers, which was video-recorded, supported a better understanding of the nature of the productions and their meanings beyond the situation of playful activity. This article is limited to present the analysis of only one of these investigated cases - a boy with hearing loss, who struggled with the organization of mathematical algorithms.

Keywords: children calculate; subjective configurations; mathematical learning; learning and inclusion 


\section{Introducción}

El estudio presentado en este artículo tiene como objetivo investigar y analizar la producción de significados, sentidos subjetivos y posibles indicadores de creatividad en las producciones de esquemas mentales matemáticos de un grupo de niños en situación de riesgo, como seres matemáticos. Apoyado en la Teoría de la Subjetividad (González Rey, 2008) y en la Epistemología Cualitativa de Fernando González Rey (González Rey, 2002, 2014), buscamos extender nuestro análisis de las producciones de esquemas mentales en actividades lúdicas más allá de la Teoría de los Campos Conceptuales de Gérard Vergnaud (1998), buscando describir y comprender, por un proceso interpretativo y de teorización, las producciones de registros matemáticos de los niños en situación de exclusión social.

Además, nos apoyamos en la categoría "ser matemático" que es propuesta a partir de la posición epistemológica de que el aprendizaje matemático escolar no debe constituirse en la asimilación mecánica de procedimientos operatorios como prevalece hoy en nuestras escuelas. La epistemología que sustenta el concepto "ser matemático" (Muniz, 2001) considera al sujeto que aprende como efectivamente productor de conocimientos y saberes matemáticos, dentro o fuera de la escuela, en situación de éxito o fracaso escolar. O sea, incluso en situación de fracaso y exclusión hay aprendizajes, pues todo sujeto aprende.

Esa perspectiva epistemológica reconoce que el aprendizaje y la construcción de saberes matemáticos no son lineales, tampoco exenta de errores. Los caminos tortuosos, los aparentes retrocesos y los errores en la búsqueda de la matematización pueden dar acceso al educador, así como al investigador, la comprensión de los complejos procesos (conceptualización, construcción y validación de procedimientos, desarrollo del lenguaje y registros, argumentación y prueba) que determinan el aprendizaje matemático, no siendo válida, por tanto, en esta perspectiva, la postura de validar una producción de un ser matemático como universalmente válida y cierta, como verdad matemática. Al final, lo que nos interesa es la estructuración (incluso si esta es siempre parcial) y la comprensión (lo que requiere un esfuerzo interpretativo) de la construcción de conceptos matemáticos (conceptualización) y de procedimientos resolutivos, aun incompletos, sin valor para generalización o no, incluso, que matemáticamente sean errados, pero de alto valor para la formación de los pensamientos del ser matemático en fase de constitución.

Teóricamente, el ser matemático es aquel que aprende y que desarrolla procesos cognitivos, esquemas mentales propios para la superación de dificultades, de enfrentamiento de desafíos, que produce procesos resolutivos para situaciones-problema matemáticos, que cree en su propia capacidad de generar nuevos procedimientos para situaciones inéditas. Aunque tales procesos matemáticos, generados por el sujeto para resolver problemas, tengan validad solamente local, sin valor general o científico, esos procesos pueden revelar capacidades cognitivas articuladas a la construcción de conceptos y procedimientos. En análisis microgenéticos, podemos explicitar esquemas mentales (Vergnaud,2009) que dan sustentación 
a la estructuración del pensamiento matemático presente en la actividad matemática del niño o joven, revelando conceptos, hipótesis, procedimientos, lógicas propias del individuo que busca elaborar una solución de una situación dada, así como las estrategias y valores de su validación.

La negación de la existencia ontológica de un ser matemático en cada uno que aprende y se desarrolla es característica de contextos educacionales en que los conceptos de correcto y errado de las producciones matemáticas de los niños y jóvenes están colocados de forma equivocada. En el contexto del aprendizaje matemático, en la búsqueda de la contribución del desarrollo de las capacidades matemáticas, mas importante que juzgar una producción estrictamente como correcta o errada, sea en relación a su valor práctico o científico, debería el educador pautar su acción pedagógica por la valorización de los procesos de aprendizaje que requieren la búsqueda de la comprensión de los procesos.

Así siendo, en la perspectiva de la categoría de ser matemático, el educador matemático (aquel que promueve la mediación o interviene en los procesos del aprendizaje matemático) no debe limitarse, en el inicio del aprendizaje de nuevos conceptos-procedimientos, a juzgar la validad de los procesos de producción matemática de cara del conocimiento científico universalmente sistematizado. Cabe al profesor sumergirse en un esfuerzo de interpretación de las lógicas inherentes a las producciones matemáticas de aquel que está en proceso de aprendizaje, en un importante cambio epistemológico: no juzgar la producción del niño apoyado en los algoritmos matemáticos ortodoxos, midiendo la producción del niño cuanto se aproxima o se distancia de las verdades matemática puestas como inmutables. Debería cada educador buscar comprender y participar de la construcción de los conceptos y procedimientos que, en el desarrollo de aquel que aprende, revelarse inestables, provisorios, no validados, con veracidad y validad solamente locales y circunstanciales. Captar, comprender, valorizar, socializar e institucionalizar los caminos, descaminos, atajos, retrocesos antagonismos, provisoriedad, recursividad. Estos fueron nuestros objetivos (Muniz, 2009) al analizar los procesos de producción de registros matemáticos de niños consideraos en situación de dificultad por la escuela. De las producciones matemáticas, en acción, de aquel que aprende son formas de considerar que cada niño y cada joven, en la realización de tareas matemáticas, son seres matemáticos en plena movilización de saberse construidos a lo largo de sus historias sócio-cognitivas-emociones y sociales, teniendo siempre a cada nuevo desafío, revisar conceptos y procedimientos, haciendo evolucionar sus conocimientos para alcanzar nuevas y más desafiantes situaciones-problema.

Contribuciones de la Teoría de los Campos Conceptuales - TCC del psicólogo cognitivista, didacta y matemático francés, Gérard Vergnaud (1994), despiertan para un nuevo enfoque a las producciones matemáticas de los niños: lógicas y verdades localmente validadas. Si asumimos que, en el proceso de aprendizaje, las construcciones de saberes matemáticos son apenas localmente validados, eso acaba por revelarnos cuan mediocre es la escuela que se limita a atribuir la producción matemática escolar solamente como "correcto" o "errado", perdiendo la oportunidad de comprender, individualmente, los procesos de atribución de significados en relación a aquel que está por aprender. 
En la intención de revelar una capacidad intelectual para procesos de matematización presentes en la escuela de los cuales somos testigos, buscamos, discutir acerca de la estrecha relación entre hacer matemática y desarrollo de la inteligencia en los primeros grados de la educación básica, con niños considerados en situación de dificultad en aprendizaje matemático, en nuestros análisis, acaban por revelarnos cuna sagacidad cognitiva que nos lleva a considerar la presencia de procesos, inteligencias generalmente eliminadas del proceso curricular escolar.

Por tanto, el objetivo de este estudio es analizar la producción de significados, sentidos subjetivos y posibles indicadores de creatividad en las producciones de esquemas mentales matemáticos de un grupo de niños en situación de riesgo. Nuestro desafío fue promover tanto una ampliación de la categoría ser matemático como sujeto productor de sentidos subjetivos en el aprendizaje matemático, como ampliar la comprensión al respecto de los fenómenos que llevan ese sujeto a producciones diversas y complejas de configuraciones subjetivas expresas en relaciones con los objetos y las actividades matemáticas. Esa ampliación de la búsqueda de la comprensión del ser matemática como sujeto, hoy desconsiderada tanto en el campo de la psicología como en el contexto didáctico-pedagógico.

Comprender las producciones de conceptos y teoremas matemáticos, la autoimagen para el aprendizaje, la configuración de sus emociones frente a la matemática puede ser plausible a partir de la construcción de espacios e instrumentos de diálogo con los sujetos que viven producir, a testear, a rever, a reconfigurar su visión de los papeles de la matemática para su desarrollo humano en relación con su mundo social y cultural.

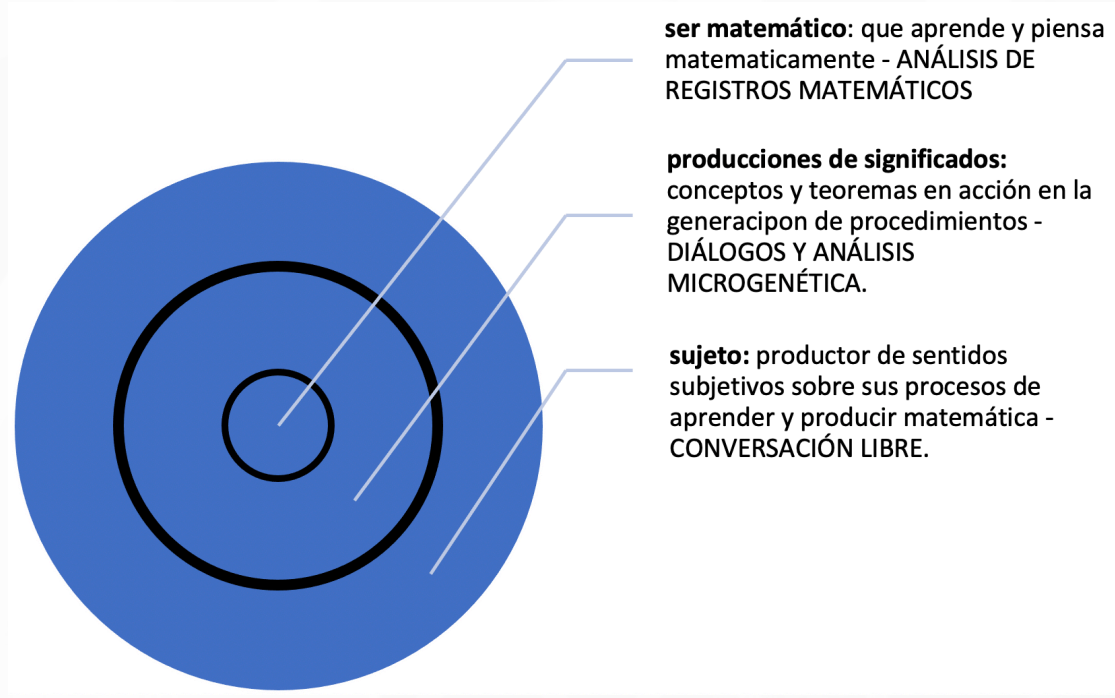

Figura 1: La constitución del ser matemático y las producciones de sentidos subjetivos 
Por tanto, hipotetizamos que la producción matemática del ser matemático es determinada, dentro de muchos factores, por su condición ontológica de sujeto, activo en los procesos de aprender, de aprender matemática, en que el sistema simbólico emocional define opciones, posiciones, caminos, intensidad de adhesión a la actividad, autoimagen, autoconfianza para superación de desafíos matemáticas y más, participa determinantemente si su emocionalidad en la relación con los objetos matemáticos, una emoción que califica los procesos de producción matemática, de aprender matemática y, en la visión del ser matemático, de sí mismo, como productor de conocimientos y sentimientos.

\section{Método y procedimientos metodológicos}

Valorizar las producciones requiere cambios en nuestras concepciones de matemática, de su producción, de su aprendizaje de su valor social y formativo. Cambios muchas veces no elementales, pues vienen al encuentro a nuestras formaciones académicas. Todavía es justamente el profundizar sobre tales producciones, el diálogo con los niños autores, el intercambio con colegas que nos permitirán una discusión más amplia de orden epistemológica que trata de la escuela como espacio de producción de saberes y no solo de consumo de conocimientos escolares.

En la perspectiva de la investigación cualitativa defendida por González Rey (2002, 2014), asumimos desde el inicio que investigar es producir conocimiento, y que es reflejo de las formas y procedimientos en que se constituyen las relaciones entre el investigador y los sujetos productores de información. En este sentido, se buscó en los procedimientos dar valor y captar producciones y sentidos de los diferentes sujetos, con el recurso esencial de variedad de procedimientos de captación de las acciones, registros, pensamientos, sentimientos presentes a lo largo de sus producciones matemáticas.

Así, nuestro enfoque está apoyado en González Rey, en la búsqueda de la confrontación de diferentes producciones obtenidas por intermedio de la diversidad de recursos y procedimientos, donde el diálogo entre investigador y sujetos matemáticos, fue la columna vertebral del método, una vez que los sentidos subjetivos de las producciones, aliados a la propia historia sócio-educativa de cada uno, con sus experiencias dentro y fuera de la escuela, relatos de los propios niños y por sus madres, con conversaciones libres videograbadas, después de 10 meses de convivio en talleres de matemática lúdica, pudieron permitir la captación más cualitativa de las producciones matemáticas y sus sentidos subjetivos para el sujeto y su grupo comprometidos en la actividad.

Las muchas y posibles posturas ante el objeto de investigación, la construcción conceptual, epistemológica y teórica, así como la postura política delante del campo de investigación, en especial, referente a la escuela y sus agentes, determinan ciertamente la naturaleza del conocimiento en producción, así como la constitución de la relación entre la academia y la universidad.

Dentro de las múltiples posibilidades de concebir la investigación, objetivando comprender la naturaleza epistemológica de la dificultad en el aprendizaje matemático escolar de la educación básica, 
articulando el análisis microgenético con procesos metacognitivos. Esto requirió el desarrollo de la investigación-acción con permanencia constante en el espacio escolar y el convivio profundo y fecundo con los estudiantes y profesores.

El estudio fue realizado durante 10 meses, a lo largo del año 2014, construyendo un taller de matemática lúdica, para un grupo de niños en situación de riesgo que participan regularmente del Centro de Vivências do CRAS (Centro de Referência de Ação Social de Alto Paraíso de Goiás). Los sujetos participantes de la investigación son niños de los primeros años de la Educación Básica (Ensino Fundamental en Brasil), 11 niños entre 6 y 11 años, entre $1^{\circ}$ y $5^{\circ}$ grado de Educación Básica en la escuela pública de Alto Paraíso de Goiás, siendo 6 niñas y cinco niños. Las características del grupo participante son:

- Habitante del interior del estado de Gioás-Brasil.

- En situación de riesgo, provenientes de familias de bajo ingreso.

- Ninguno vive con el padre biológico.

- Estudiantes de primeros grados de Educación Básica en la escuela pública.

- Frecuentan el Centro de Actividades del CRAS (Centro de Referencia de Asistencia Social), que ofrece acompañamiento de los quehaceres de casa, talleres como teatro, circo, capoeira, en el año 2014, Taller de Matemática Lúdica. La inscripción en los Talleres es espontánea, de acuerdo con el interés- además de las actividades, el CRAS provee refrigerios diarios para los niños participantes.

- Mas del 90\% de las madres de los niños no tienen la Educación Básica completa. Trabajando en ventas, servicios caseros o ama de casa. Las madres se observan muy dedicadas a la educación de sus niños, buscando estimularlos para el estudio y realización de actividades que favorezcan el desarrollo de variedad de habilidades. Todas acompañan de cerca la vida escolar de los niños, teniendo presencia constante en la escuela y en las actividades del Centro de Actividad.

\section{Resultados y discusiones}

El estudio fue realizado con un grupo de once niños en proceso de escolarización, de los primeros años de Educación Básica, todos en situación de riesgo social, en la región de Chapada dos Veadeiros, importante APA (Área de Protección Ambiental) del Centro-Oeste brasileño. El grupo era atendido

2 El Ensino Fundamental en Brasil es de 9 años y se divide en dos: Ensino Fundamental $I$ o años iniciales comprende del $1^{\circ}$ al $5^{\circ}$ y el Ensino Fundamental II o años finales comprende el $6^{\circ}$ al $9^{\circ}$. 
por la Secretaría de Acción Social, en contrapartida de la ida a la escuela, donde creamos, por medio de la investigación un ambiente ludo-matemático, con actividades, materiales y juegos que favorecían el aprendizaje matemático previsto en el currículo escolar. En este texto traemos el caso de Rochinha ${ }^{3}$ (seudónimo), por tratarse de un niño que, además de estar en situación de riesgo social, es deficiente auditivo, lo que dificulta tanto su comprensión de los conceptos y procedimientos matemáticos, como representa dificultad de comunicación de los pensamientos matemáticos de la escuela. Hay datos en su registro en el proyecto municipal que Rochinha tiene dificultades escolares, sobre todo en el aprendizaje matemático.

\section{Rochinha, en la búsqueda de la alfabetización matemática.}

Rochinha, con siete años de edad, estudia el segundo grado, desde el inicio de las actividades se observa como un niño alegre, interesado en participar, demostrando gran curiosidad por los materiales lúdicos y las actividades de juegos matemáticos. Incluso no siendo el mas pequeño del grupo, es el que tiene menor estatura. Le gusta mucho realizar juegos corporales, de luchar, correr, siempre nos recibe con una bella sonrisa, desde el momento de nuestra llegada cada tarde.

Es necesario destacar que las actividades matemáticas que permean los juegos son de grados de dificultad que crecen, o sea, conforme el juego avanza, las dificultades en las resoluciones de problemas, los contextos y el orden de la grandeza de los números involucrados aumentan cada vuelta.

Siempre presente en las tardes de los jueves en el Centro de Actividad, muchas veces, observamos su alegría por la participación y su curiosidad acaban siendo sustituidas por el aislamiento en el rincón del aula, con la negativa de continuar participando y con el rostro cerrado. Tal actitud nos preocupa, y busco aproximarme a él, pero él se niega a hablar porqué su negativa a participar.

Rochinha evita cualquier aproximación o tipo de ayuda, no queriendo decir el porqué, dejó de participar, tampoco deja entrever al grupo sus dificultades. Niega asimismo cualquier aproximación de su hermana, participante del grupo, mayor que él, Tania con 10 años y asiste al quinto grado de Educación Básica.

Las actividades matemáticas no presentan linealidad ni de contenidos, de conceptos, tampoco de dificultades en los juegos. Como el grupo es bien heterogéneo, se buscó en la planificación y desarrollo una variación de esas actividades, proveyendo la movilización de conceptos de formas recursivas, buscando envolver un mismo concepto en diferentes juegos, así como variar el grado de dificultad dentro de un mismo juego.

\footnotetext{
$3 \mathrm{El}$ nombre de los niños es ficticio buscando preservar la identidad de los niños participantes. Los nombres son escogidos teniendo por base trazos de la personalidad de cada participante: Rochinha, el cineasta y cerrado. En el original Alfabetização Matemática.
} 
Con el paso de las actividades, con aproximación gradual, gané la confianza de Rochinha y vimos surgir la construcción de una relación dialógica, fue cuando podimos tomar conocimiento de sus procesos de aprendizaje, sus sentimientos en relación a la matemática, y como se percibe en esas relaciones favorecidas por las actividades lúdicas. Es interesante resaltar que la explicitación de las potencialidades, de los limites y de sus dificultades es revelada solamente en el desarrollo de los juegos, de los registros, de los intercambios presentes en la actividad, siendo que, en algunos momentos fuimos buscados por él para colocar sus dudas, dificultades o incluso, como hicieron algunos niños (sobre todo en relación a la división), demandando que, en el Centro, trabajásemos contenidos escolares con los cuales están teniendo dificultad o curiosidad. En ningún caso se llevaron tareas para hacer en el Centro.

Esas dificultades observadas a lo largo de las actividades realizadas por Rochinha serán ahora presentadas y analizadas, pero siempre tomando en cuenta le contexto del juego matemático, las exigencias de conceptos, procedimientos y registros. Después de discutir una serie de producciones de Rochinha, vamos a traer un poco de sus propias palabras sobre sus procesos subjetivos, una ves que, en sus registros, como veremos, hay fuertes indicadores de configuraciones subjetivas en el complejo proceso de su aprendizaje matemático. En un momento posterior, damos voz a la madre del niño, para situar histórica y afectivamente el desarrollo de él, lo que puede ayudarnos, por medio de la conversación, confirmar o no hipótesis sobre el proceso de configuraciones subjetivas.

\section{Estructura del número: lectura, comprensión y registro.}

Como se dijo anteriormente, hay una percepción de nuestra parte, desarrollada en el trabajo empírico, que hay fuerte tendencia de evasión de la actividad, cuando Rochinha se enfrenta con números de mayor orden o cuando hay en la actividad lúdica una propuesta de lectura,interpretación, construcción de procedimientos operatorios con registros que requieren la necesaria comprensión de la estructura del número, incluso que este sea un número natural.

Fueron muchas actividades que movilizaron la estructura del número y sus consecuentes interpretaciones y lecturas, dentro de ellas están: bingo, juego de la alfombrilla, juego de la vendimia, carreras, juego de memoria, entre otros. Pero fue en el juego de las fichas escalonadas, aplicados el día 13 de noviembre que pudimos efectivamente constatar el nivel de dificultad de Rochinha sobre la comprensión y producción de significados de la estructura del número.

Al trabajar con las fichas escalonadas, pudimos ver, en situación de necesidad de lectura, interpretación y comparación que números mayores que 100 generaban grandes dificultades, tal como el número 789 y Rochinha lee: "setenta y ocho... nueve" o aún 1994 como "diecinueve noventa y cuatro" revelando que posiblemente su alfabetización matemática ha quedado restricta a una centena exacta. Por otro lado, él no se niega a leer y a comparar y producir un esquema mental que 
está sustentado en sus construcciones previas: lee los números de dos en dos dígitos, limitándolos, en su composición, a las decenas. Hay momentos en que su lectura es "ciento y cuarenta y cinco... ocho" para el número (1458).

En la conversación con su madre, durante el proceso, ella afirma que nunca la escuela había llamado la atención para tal problema, a pesar que ella ha ido diariamente a la escuela y ha acompañado las tareas. Cabe desde ya informar que Rochinha reprobó el año lectivo en 2014, el segundo grado, en pleno ciclo de alfabetización, pues no conseguía realizar las actividades matemáticas escolares.

La actividad lúdica con las fichas escalonadas ayuda mucho a los niños a percibir las estructuras del número y acaban por revelar sus hipótesis sobre tales estructuras y consecuente lectura y escrita interpretativa de las cantidades. Como vemos a continuación en el registro de Rochinha en el juego de las Fichas Escalonadas, acabamos por comprender un poco más de sus producciones.

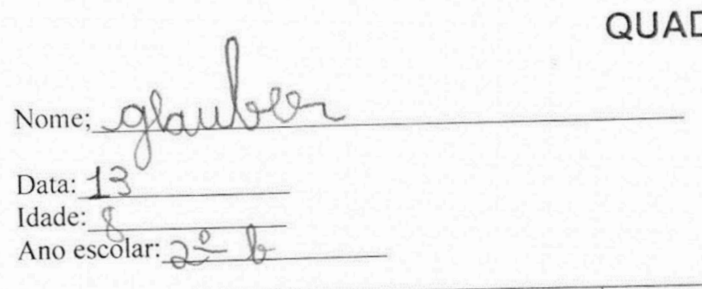

\begin{tabular}{|c|c|c|}
\hline Número & Composição & Escrita \\
\hline 124 & $100+20+4$ & cento evinte e quatro \\
\hline 34 & & \\
\hline$\frac{77}{805}$ & $\begin{array}{l}70+7 \\
800+5\end{array}$ & $\begin{array}{l}\text { petinto \& set } \\
\text { vitos entos cince }\end{array}$ \\
\hline $\begin{array}{l}8408 \\
9003\end{array}$ & $800+40+8$ & oets les \\
\hline & & \\
\hline
\end{tabular}

Figura 2: Descomposición y escritura de los números revelando la escritura como se lee

En el cuadro número-composición-escrita, siendo el número dado por el investigador, la composición dada por el uso de fichas y la escritura una composición a partir de las lecturas de las fichas utilizadas. Al solicitar en el juego la composición con las fichas el número 848, escribe "8408”, o sea, por nuestra interpretación “ochocientos y cuarenta y ocho", o sea,

"Ochocientos y cuarenta $\rightarrow 840+$ ocho $\rightarrow$ 8", sintetizando: 
$8 \rightarrow$ ocho, así tenemos “ochocientos y cuarenta y ocho” $=8408$.

Tal análisis interpretativo es reforzado en la línea siguiente con el próximo número, 903, o sea, novecientos y tres, y su producción, la cual acaba por abandonar queda como:

900

$3 \rightarrow 3$, así tenemos $9003=$ novecientos y tres.

En la actividad con las fichas escalonadas, para Rochinha, la composición del número y consecuente escritura-lectura implica incluso la no sobreposición de las fichas de forma a producir 903 y queda solo con la sobreposición permaneciendo la ficha 900 sobrepuesta a la ficha 3, lo que genera 9003, siendo, de acuerdo con la hipótesis de escritura de Rochinha y en acuerdo con su escucha. "novecientos y tres" $=900+3$.

Ese análisis e hipótesis ganan fuerza teórica y lógica cuando traemos las palabras de María, madre de Rochinha, y del propio niño cuando él hace críticas a las posturas de la profesora ante sus dificultades, culminando, incluso en la reprobación del año escolar 2014.

Ahora veremos que esa cuestión de la forma de apropiación de la estructura del número por Rochinha implica consecuencias, también en los procesos de generación de procedimientos operatorios e, incluso, dificultando la comprensión de los algoritmos ortodoxos impuestos por la escuela desde el proceso de alfabetización.

\section{Estructura del número: implicaciones para la producción y registro de procedimientos operatorios.}

Más allá de la cuestión más estricta de la producción de escritura y lectura interpretativa de los números, constatamos, en nuestros análisis interpretativos de sus producciones de registros matemáticos, que la no apropiación debida de la estructura de los números por Rochinha, acaba por dificultar la producción de procedimientos eficientes.

A lo largo del proyecto fueron muchas las situaciones en que los juegos requerían de los niños participantes la producción de procedimientos operatorios, como en el sendero, el mercadito, en la alfombrilla, en el juego de los platitos con la división, entre otros. Así, en las producciones a continuación, en las cuales tenemos las operaciones $1847+2356$ y $145+382$, se ve que incluso con números mayores, pero con el mismo número de orden, como en la suma, Rochinha no tuvo dificultad, pues el posicionamiento espacial es mas evidente, acabando por quedar un orden abajo del orden, llevando al éxito al sujeto. 


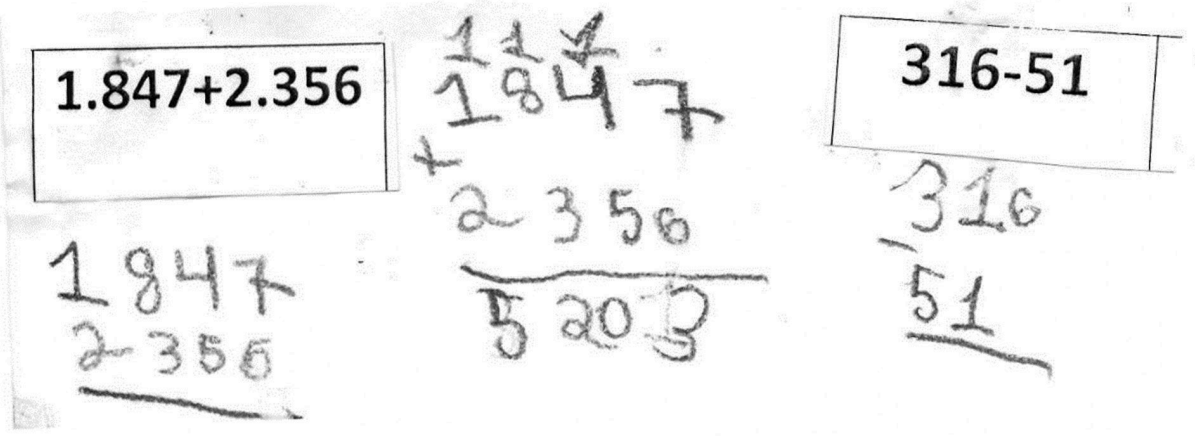

Figura 3: Cuestión de la espacialidad influenciando el posicionamiento y construcción de procedimientos.

Sin embargo, en el caso de 361 - 51, una operación con menor orden de grandeza (no discutiremos ahora el hecho de que la misma implica un desagrupamiento decimal) vemos la dificultad de localización espacial de Rochinha con los dígitos, acabando por quedar el 5 debajo de las 3 centenas, lo que inviabiliza la realización de la operación, una vez que da la impresión de que hará 300 - 500. Así, Rochinha la abandona y no marca el resultado en la cartelera del bingo numérico. La misma dificultad de orientación espacial y posicional con los dígitos y sus órdenes, ocurre en el juego del Bingo. En ese juego, son distribuidas operaciones en una hora cuadriculada, en las cuales cada jugador debe recortar, operación a operación. Ahí el investigador a lo largo del juego, uno a la vez, sortea una operación que debe ser pegada en una hora provista en el juego por el investigador. Al resolver en la hora la operación, el jugador intenta identificar ese resultado en la cartelera. En caso que lo tenga, marca el número con una " $\mathrm{X}$ ".

En un momento del juego, Rochinha al realizar $279+15$, tiene dificultad en los posicionamientos de orden, lo que lo lleva a totalizar 942, perdiendo la oportunidad de marcar un punto, pues ese total no se encuentra en las carteleras (observamos que no encuentra un número en la cartelera, puede, para un niño en juego, interpretar como un posible error en su cuenta, pero esto no ocurre con Rochinha):

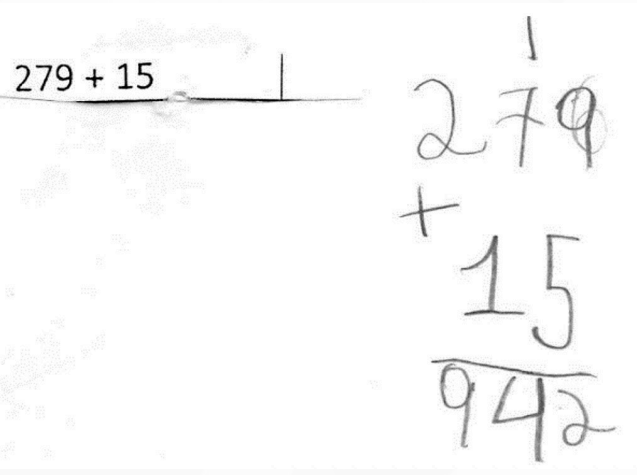

Figura 4: pregunta de dificultad de especialidad como obstáculo en la producción matemática. 
Vemos el esquema mental explicitando en forma de teorema en acto (Vergnaud, 2009). Suma $9+5=$ 14, quedando 4 unidades, promoviendo una decena y sumando las decenas, totalizando nueve decenas y allí, aparece nuevamente la cuestión espacial. Colocando las dos centenas que sobran a la derecha del total y no a la izquierda, revela no tener conocimiento de que este "dos" es de centenas. De esa forma, la cuestión espacial de posicionamiento se articula y es sumado a la cuestión de no comprensión de números a partir de 200. Esto ocurre nuevamente cunado un número tiene centenas y otro no. En la misma hora de producciones de registros, vemos otro caso, en el cual ambas partes tienen centenas y en este, Rochinha no presenta cualquier dificultad.

En este punto del análisis y producción de interpretación, cabe la pregunta: hasta qué punto Rochinha tienen conciencia de la naturaleza de sus dificultades que están necesariamente articuladas a la cuestión posicional y a los significados de la estructura de los números en órdenes y clases, así como su comprensión entre valores posicional y decimal. En esta última producción vemos que la noción de valor decimal se revela muy avanzada, quedando nuestro indicador de dificultad direccionado para la cuestión posicional.

Un hecho importante revelado en el proceso interpretativo es que, desde el inicio de las actividades, Rochinha busca producir respuestas, se compromete en una actividad cognitiva importante, no abre mano de su derecho en participar y producir registros matemáticos, según sus capacidades y potencialidades, no negando la existencia de dificultades. Eso revela que, en las actividades en que abandona el juego, se aleja y queda visiblemente aburrido, puede ser el reflejo de una toma de conciencia de que las situaciones no pueden ser mas controladas por él y no se ve capaz de producir una solución, pues los valores a partir de un momento de juego están más allá de su nivel de aprendizaje, sobre todo en relación a la estructura de los números.

Entonces, el diálogo para la interpretación y comprensión de esos procesos complejos y no lineales requieren, coadyuvando con la perspectiva de la Epistemología Cualitativa de González Rey, la constitución de un ambiente impregnado por el diálogo entre el sujeto y el investigador, en la búsqueda de elementos de interpretación que favorezcan que la producción de conocimiento produzca las informaciones más próximas posibles de ese proceso psicológico que el propio sujeto epistémico no tiene conciencia. Así, la articulación de las palabras del sujeto como los registros producidos (así como la explicitación de esquemas mentales), puede proveer importantes herramientas de análisis para la explicitación de producción de significados y sentidos subjetivos.

Una diferencia de orden epistemológica de este estudio en comparación a los anteriores en el campo de la educación matemática es que, al apoyarnos en la Epistemología Cualitativa, no nos limitamos solo a la explicitación de los esquemas mentales (Vergnaud, 2009) en la producción de registros de esos niños, sino que buscamos ampliar las fuentes de producción de informaciones para nuestras interpretaciones y búsqueda de indicadores. A partir de la identificación de los esquemas explicitados en forma de conceptos y teoremas en acción (Vergnaud, 2009), buscamos articular la comprensión de 
esas producciones a la percepción mas amplia de la propia constitución del sujeto, como sujeto social y emocional, entrelazando esos conceptos y teoremas a la propia comprensión de su historia constitutiva.

\section{Palabras de Rochinha como sujeto matemático.}

Así, partimos ahora para las palabras del propio Rochinha que nos revela elementos importantes de su propia imagen constitutiva y nos hace comprender mejor sus producciones matemáticas y el complejo proceso de aprendizaje matemático y desarrollo humano. En el inicio de la conversación videograbada, Rochinha habla de sus preferencias en la escuela:

\section{(...)}

Investigador: Ok. Y en la escuela, ¿qué te gusta de la escuela?

Niño: A mí me gusta el recreo.

I: El recreo. ¿qué tiene de bonito los recreos que te gusta tanto?

$\mathrm{N}$ : Los juguetes.

I: ¿Cuáles juguetes?

$\mathrm{N}$ : Hay uno grande y allí nos subimos en él y nos quedamos arriba.

I: Y además del recreo, ¿qué te gusta?

N: Matemática.

I: Ah, ¿te gusta?, ¿'sólo porque yo estoy aquí?

N: No.

I: ¿No?, ¿siempre te gustó la matemática?

$\mathrm{N}$ : Antes no.

I: ¿Antes no?, ¿`ómo es que te comenzó a gustar la matemática?

$\mathrm{N}$ : Cuando tú me diste clases

I: ¿Ah sí?, ¿ُfue la clase que te di?

N: Sí.

Los placeres están fuera del aula, están en la recreación, en la "no clase". Al principio, el gusto por la escuela no está vinculado a los contenidos del componente escolar. Cuando es provocado a decir su preferencia, él cita inmediatamente Matemática, pero es por relación afectiva que surgió solamente el año pasado a causa de las experiencias vivenciadas en el Centro en función de nuestro Proyecto. Así podemos constatar inicialmente que, de alguna forma, las actividades lúdicas en el Proyecto influenciaron la relación con el área de conocimiento, lo que fue fundamental para el delineamiento de las estrategias educativas en el proceso.

Es interesante notar que Rochinha ve esa experiencia como "clase", aunque las actividades no ocurran en la clase, no había pizarra ni libro, cuaderno formal, tampoco evaluaciones, pero desarrollado siempre por medio de actividades lúdicas y juegos. Sobre su visión de lo que era propuesto y lo que se desarrollaba durante las actividades él expone: 
I: ¿Ah sí?, y allá en el CRAS, allá en el Centro, ¿qué hacías de matemática?

$\mathrm{N}$ : Jugaba.

I: ¿El que jugabas?, ¿qué tipo de juego era?

$\mathrm{N}$ : Había unos carritos allí pasaba en los números, jugaba de unas cosas en el último ganaba una pulsera.

I: ¿Pero era solo por el premio que gustaba de la clase de matemática?

N: No.

Así, en el Centro, la visión de realización de la actividad matemática está anclada en el juego y en la premiación. El juego que caracteriza las vivencias matemáticas en el Centro es precisamente el elemento de placer experimentado en la recreación dentro de la escuela. En cuanto al cuestionamiento de su placer por ciertas actividades está en función de poder ganar ciertos premios en los juegos, Rochinha lo niega, o sea, posiblemente el placer está asociado con la actividad y experiencia vivenciada en sí. Además de esto, levantamos la hipótesis: si la afectividad no estaría asociada también al hecho de que la experimentación, en esas actividades propuestas y mediadas, favorece la superación de desafíos y dificultades, revisando sus procesos de aprendizaje matemático. Así preguntamos cómo fue, en ese mismo período, la matemática en la escuela:

I: Y tu, ¿cómo era la matemática el año pasado en la escuela?

$\mathrm{N}$ : Era buena

I: ¿En la escuela como te fue?, ¿`ómo era en aquella escuela que tu estabas? Dime un poco de eso.

N: La profesora siempre salía del aula, ella iba para otra escuela y se quedaba allá.

I: ¿Y que te quedabas haciendo en el aula?

$\mathrm{N}$ : Copiando.

I: Ella escribía en la pizarra, explica como había que hacer

$\mathrm{N}$ : Ella escribía en la pizarra y dejaba una tarea, tenia que hacer la tarea y después copiar la pizarra. Entonces ella iba y salía y nosotros con la dificultad y entonces esperábamos que ella llegara.

I: Y cuando ella llegaba, ¿qué hacía?

$\mathrm{N}$ : Explicaba.

I: ¿YY tú entendías?

I: ¿Siempre?

N: Sí

I: Ok. ¿Te gustaba hacer los deberes de matemática em aquella escuela?

N: Sí.

I: ¿Cuál fue tu mayor dificultad en matemática el año pasado? Eso que cuando tu llegabas a esa parte y decías, ¡ay Dios mío!, ¿qué era?

N: Yo llegaba y hablaba, ¡ah no esa actividad no! Entonces ella iba donde mí, tomaba el cuaderno y entonces escribía y me explicaba.

$\mathrm{N}$ : En ese momento tenía que quedarme copiando y no salía para el recreo. 
Fue una sorpresa para nosotros la respuesta inicial de que la experiencia con la matemática había sido buena, más aún considerando que fe reprobado ese año justamente por causa de la matemática. Cuando insistimos a hablar más del asunto, comienza por expresar que las actividades escolares se sintetizaban en la realización de copiar de la pizarra y destaco la permanente ausencia de la profesora del aula. Incluso acompañando la vida escolar, sabiendo de la alta desmotivación por la escuela el año pasado, el afirmó que le gustaban las actividades y que entendía las explicaciones, sin embargo, la profesora no estaba presente a lo largo de las actividades, permanecido fuera del aula, regresando solo para poner la respuesta correcta en la pizarra. Su no salida para el recreo era utilizado por la profesora como forma de control al grupo en la realización de las actividades. Esa ausencia de la profesora del año pasado en el aula queda resaltada en otra parte de su diálogo:

I: Ok. ¿Y este año como te va?

Niño: Bien.

I: ¿Por qué mejoró este año?

N: Porque es otra profesora y ella explica más incluso, ella se mantiene en el aula y solo sale de vez en cuando. Entonces ella se queda a toda hora en el aula.

Al preguntarle a lo largo de la conversación sobre lo que la profesora del año debería cambiar, él refuerza ese asunto del abandono del aula de clases:

I: Si te encontraras con la profesora del año pasado, de la otra escuela, si pudieras llegar y darle un consejo de como podría mejorar sus clases. ¿Qué le dirías?

$\mathrm{N}$ : Que ella tiene que mejorar el trabajo en matemática, portugués solamente.

I: ¿Sólo eso?

I: ¿Y cómo podría mejorar en matemática?

$\mathrm{N}$ : Que se quedara dentro del aula.

I: Que ella se quedara dentro del aula. ¿Y haciendo el qué adentro del aula?

N: Explicando.

I: Explicando...

$\mathrm{N}$ : Y que de vez en cuando saliera.

Sobre sus recuerdos acerca de las mayores dificultades matemáticas tenemos:

P: ¿Pero no recuerdas con qué matemáticas tuviste dificultad?

C: No.

P: ¿Trabajar con números, saber escribir los números?

C: Sí.

P: ¿Leer los números?

C: Sí. 
P: ¿Y sabías hacer las operaciones?

C: Más o menos.

$\mathrm{P}:$ ¿Qué operaciones conocías más?

C: Portugués.

$\mathrm{P}:$ ¿Operación matemática?

C: Fue ... lo olvidé.

P: Oh, ¿lo olvidaste? ¿'Te gustó sumar?

C: ¿Ah?

P: Suma, ¿cuentas de sumar?

C: De suma era más fácil para mí.

$\mathrm{P}:$ ¿Y de resta?

C: Más o menos.

$\mathrm{P}:$ ¿Y viste la división?

C: Sí.

$\mathrm{P}:$ ¿Y qué te pareció la división?

C: Muy difícil

Inicialmente Rochinha dijo que no recuerda cuales eran sus dificultades en Matemática, pero luego del cuestionamiento del investigador, él acaba por revelar que el trato con los números era su dificultad central, como vimos en el análisis de sus actividades. Tanto así que al ser preguntado sobre las "operaciones que mas sabía" él dice que era "Portugués", pero en cuanto a las operaciones matemáticas, ni sabía decir cuales eran. Sin embargo, su facilidad era la suma, siendo la más difícil, sobre todo la división, lo que es reforzado en los análisis de sus producciones. Sobre su olvido puede tener dos significados: (1) olvido, pues son procesos sin sentido, sin posibilidad de tejer una lógica de explicitación de las dificultades; (2) olvido que se relaciona al disgusto.

I: ¿YY allá en el Centro de actividad había división? ¿te acuerdas que estaban los platitos? N: Sí

I: Entonces, ¿Cómo fue eso allá en el Centro?

$\mathrm{N}$ : Estuvo bien, tomaba los platitos y los entregaba, hacía dupla.

I: Aja

$\mathrm{N}$ : Uno iba con uno, otro iba con otro y otro iba con otro

I: Y, ¿pudiste hacerlo?

$\mathrm{N}$ : Aja, sí.

Ya en el Centro, las actividades de división no revelaron la misma dificultad, una vez que ellas se apoyan le la construcción conceptual y en el desarrollo de procedimientos con apoyo en representaciones concretas, lo que favorece articular el reparto con el conteo dividido igualmente en el plato, con la construcción gradual de significados, aunque esos procesos no lo llevasen a la articulación con la 
estructura formal y abstracta de la división exclusivamente como algoritmo escrito. Sin embargo, él revela que en las experiencias realizadas en el Centro pudo avanzar en lo que se refiere a su mayor dificultad, es decir, el trabajo con los números grandes.

I: La página 21, ¿podía encontrar en el libro la página 21?

N: Sí

I: ¿Y la página 425?

N: Sí, podía.

I: Ah, ya, sí podías. ¿Y si fuera la página 1426?

N: No, no podía.

I: No. ¿Y hoy si puedes?

N: Sí.

I: ¿Encontrar el 1000?

N: Sí.

Hoy él puede, sobre todo después de trabajar en juegos con las fichas escalonadas, efectuar la construcción de significados con números con 3 o más orden, lo que era incluso dificultador para el desarrollo de procedimientos operatorios.

¿Quién es Rochinha para maría?... Visión maternal reveladora.

La visión de la madre aquí es importante en el sentido de que a lo largo de la conversación, nuevos elementos interpretativos e hipótesis pueden ser revisados o confirmados, a partir de elementos psicológicos traídos por ella que tiene una visión global del desarrollo de nuestro sujeto. Resaltamos que las palabras de la madre retratan solamente una imagen que ella construyó del sujeto, no siempre respondiendo a la realidad.

María no tiene formación ni de la Educación Básica, pero es trabajadora, luchadora por la manutención de sus hijos, y más que eso, busca garantizarles derechos que le fueron negados en su infancia y juventud, siempre acompañando el desarrollo de los hijos y estimulándolos para los estudios.

Sus palabras iniciales trazan la visión que tiene de Rochinha como:

Investigador: ¿Quién es para ti Rochinha?

Madre: Ah, es un niño muy especial, mi hijo es dulce. A veces tiene unos problemitas, pero es maravilloso. Es inteligente, trabajador, le gusta hacer las tareas bien hechas, es cuidadoso.

Esfuerzo y motivación son puntos de destaque en la caracterización de Rochinha, siento que no interpretamos las tareas como algo necesariamente relacionado a las actividades escolares, pero actividades de forma general. Así vemos un niño que disfruta presentar sus resultados con esfuerzo. 
Sobre el aspecto “inteligencia” pedimos que María explicase esa comprensión.

I: Usted habla que él es muy inteligente, ¿cómo percibe esa inteligencia en él?

M: Porque todo lo que hace lo hace solo, se puede desempeñar bien con las cosas que el comienza. Este año gracias a Dios él no tiene dificultades, todo para él está más fácil. Todo lo que él hace lo hace sin decirme: "Mamá, ayúdame. Fulano, venga acá”. No, él desempeña bien su papel de hijo y estudiante, en la sociedad le está yendo bien, gracias a Dios.

Así, la inteligencia expresada por María está asociada a la autonomía, a su capacidad de realizar cosas, resolviendo por sí los desafíos inherentes a las actividades. Pero la cuestión de análisis para nosotros es sobre las dificultades, en especial el hecho de la reprobación del segundo grado en 2014.

I: El año pasado, ¿cómo fue el año pasado? El año pasado él participó de las actividades del CRAS de matemática, ¿verdad? ¿A él le gustaba?

M: De la actividad allá sí le gustaba

I: ¿Qué decía el de las actividades?

M: El le gustaba las actividades con el profesor Cristiano, al él le gustaba mucho, pero en la escuela no le gustaba.

I: ¿Por qué cree que a él no le gustaba la escuela?

M: Él decía que todo mundo hablaba mal, porque él tiene problema auditivo.

I: Ah, ¿él tiene problema de audición?

M: Sí.

I: ¿Fue diagnosticado?

M: Sí.

I: ¿Y que sucedía en la escuela?

M: Entonces no le explicaban bien a él, hablaban muy bajo, entonces él no escuchaba y terminaba perdiéndose todo, la tarea, las explicaciones, hasta que él ni pasó de grado, reprobó el año pasado. Pero este año esta yendo bien, gracias a Dios.

I: ¿En qué grado está él?

M: Está en segundo.

I: ¿Tiene siete años?

M: Tiene ocho años.

I: Ocho años.

M: Segundo grado, era para estar en el tercer grado, pero él aplazó. Entonces ahora él está con una nueva profesora, Yolanda, maravillosa, todo está yendo bien gracias a Dios, está desempeñándose bien el papel de él fue bien provechoso lo que él aprendió con el profesor Cristiano, porque él ahora hace las tareas de matemática sin necesidad de ayuda, y fue bien provechoso ese tiempo que él estuvo haciendo ese trabajo. 
Para María, Rochinha disfrutaba participar de las actividades en el Centro de Actividades e incluso, eso contribuyó para sus aprendizajes en matemática, pero en la escuela la realidad era diferente, sobre todo en función de un hecho revelado por la madre, que Rochinha tiene deficiencia auditiva y no posee prótesis, lo que dificulta la comunicación. En el año 2014 la profesora, según María, no daba la atención necesaria, incluso sabiendo de la deficiencia auditiva, haciendo con que perdiese partes de las explicaciones, lo que implica la pérdida de los significados de los contenidos. En este año, a él le está yendo bien, pues, además de los aprendizajes realizados en el Centro, la profesora está presente y se comunica con él.

Vale destacar que el investigador no estaba consciente de tal deficiencia auditiva, pero que la configuración de la realización de las actividades en el Centro, siempre en pequeños grupos, con muchos intercambios, con atendimiento individualizado, con atención uno a uno, debe haber permitido la plena comunicación de Rochinha, comprendiendo siempre los comandos y comprometiéndose en las actividades. En ningún momento la coordinación del CRAS o del Centro de Actividades informaron de tal deficiencia auditiva ni su madre, a pesar de nuestro contacto personal constante.

En este punto, retomamos el hecho de las dificultades de Rochinha con cuestiones tanto de la estructura del número como de la construcción y registro de operaciones, en que la dificultad de la especialidad, conforme apuntamos en los análisis iniciales, se revelan como obstáculo al aprendizaje matemático. Con la existencia de una deficiencia auditiva, podemos percibir que la cuestión de la especialidad no es un problema de orden cognitivo o de déficit cognitivo, pero de las propias formas de relación con el espacio y producción de registros, como ya pudimos constatar en análisis de producciones matemáticas de otros niños con deficiencia auditiva (Muniz, 2004). Las diferentes formas de especialización no pueden ser sumidas como dificultades en las producciones de registros, sino como posibilidades de producciones diferenciadas cargadas de sentidos subjetivos y como fuente de producción de configuraciones subjetivas en el quehacer matemático, lo que se ha negligenciado en los procesos educativos escolares.

Son formas diferenciadas de producciones espaciales en el trato con los números, que son consideradas por la escuela como factor de no aprendizaje matemático y redundando en la retención de Rochinha en el segundo grado escolar. No hay evidencia que la escuela profundizase sobre las producciones matemáticas en la búsqueda de efectivas causas de su dificultad en la construcción de procedimientos y registros matemáticos.

En cuanto a las percepciones de la madre en relación a las dificultades de Rochinha, sus palabras revelan que su percepción sobre sus aprendizajes matemáticos es parcial y considerando la paciencia que tiene en ayudarle no corresponde a lo que presenciamos en el Centro y en la Escuela, cuando muchas veces María estaba irritada y agresiva con su hijo por no "prestar atención" a las tareas, pero parece que esta es una historia que quedó en el pasado: 
I: $Y$ en el pasado, cuando vio que él estaba teniendo dificultades en la escuela, que no estaba motivado, ¿intentó ayudarlo? ¿Como lo hizo? Tuvo paciencia, ¿cómo fue?

M: Sí, siempre lo he hecho. Cuando veo que no puede aprender me siento, voy allá para ayudarlo a hacer las "tareas", le explico cómo es, pero cuando llegaba a la escuela, él no tenía mucha atención, entonces él no lo hacía.

I: ¿Usted lo acompañaba?, ¿qué notó?, ¿cuáles eran sus dificultades en términos de matemática?, ¿Qué notó que él no podía aprender, hacer?

M: A veces la división, no podía dividir correctamente, la resta, a veces no podía.

I: ¿Qué tipo de resta él no podía hacer?

M: La resta en general, casi todas no podía hacerlas, entonces lo que hizo más era la suma que era la que menos dificultad le daba. Pero después del curso que hizo allí, hasta ahora está bien, hace de todo y ni siquiera me pide ayuda, es muy raro que ahora me pida ayuda para hacer la tarea. Ahora lo único que todavía le cuesta un poco es que tiene que seguir hablando más alto, porque no escucha bien, entonces la profesora tiene que hablar un poco más alto.

Inicialmente las palabras de María se centran en la división, pero acaba admitiendo que la resta le imponía mucha dificultad también, pero con la participación en las actividades matemáticas en el Centro (que ella llama curso), estas fueron superadas y que este año los resultados al momento muestran que esa dificultad fue superada y Rochinha incluso ganó autonomía y motivación para las actividades matemáticas escolares.

El asunto de la deficiencia auditiva se presenta como un hecho negligenciado tanto por la escuela como por el equipo del Centro, tanto así que en ningún momento alguien evidenció ese hecho al investigador, incluso cuando Rochinha evadía las actividades matemáticas, por percibir que no podría producir respuestas:

I: Pero y la profesora del año pasado, ¿también no sabía que él tenía problema auditivo? M: Ella sabía, pero ella no le dio mucha importancia.

I: No le dio. ¿Y el personal del CRAS sabía también?

M: El personal del CRAS también sabía, todo mundo allá en la escuela sabe que él tiene ese problema.

I: Y, ¿por qué cree que él a veces el rehuía del taller de matemática? ¿Qué él no quería quedarse?, ¿por qué cree que él no quería quedarse?

M: Es porque él hablaba que creía que todos los profesores son iguales, que no iban a darle importancia a él, que no iban a darle explicaciones para él y para todo el mundo, no iban a dar una atención especial para él, tanto así que después él pasó a quedarse en las clases de una vez, ¿iverdad?, al comienzo él se escapaba de las clases. 
Así, los sentidos subjetivos producidos por Glauber asociados a la falta de atención de los profesores, pues consideraba que todos los profesores iban a negarle la posibilidad de una comunicación efectiva, o que acabó no sucediendo en las actividades de Matemática en el Centro.

Sus evasivas pueden ser interpretadas como forma de distanciamiento del sujeto en relación a la situación que ve como contexto de impasse, de imposibilidad de transponer, sin oportunidad de superación, pues no había como encontrar ayuda, visto que la ayuda y la mediación pedagógica siempre le fueron negadas en la escuela el año pasado. Si no pudo obtener ayuda y no se autoexpuso al bloqueo, es una estrategia de protegerse dentro del grupo. Al excluirse, acaba por instaurar un ciclo vicioso, una vez que, socialmente excluido, no hay como ofrecerle ayuda y, por consecuencia, el aprendizaje o se realiza, reforzando su autoimagen de no capacidad de superación de situaciones matemáticas.

Para ampliar la visión de las capacidades y dificultades de Rochinha, llevamos a una discusión a María sobre la vida de Rocbinha más allá de la escuela:

I: ¿Y en casa? ¿cuáles dificultades usted percibe que el tiene en casa, cuales son las facilidades? ¿cómo es el día a día de él?

M: Ah, a él le gusta mucho arreglar la casa.

I: Ah, el colabora.

M: El colabora, a él le gusta ayudarme a lavar ropa, ¡le gusta! Él no le gusta mucho barrer, si yo le digo: hijo, barra allí, entonces eso no le gusta. El baño: esa es otra historia, no es para él, pero voy a lavar ropa y él está allí a mi lado, tanto que solo él me ayuda a lavar ropa, los demás casi que no hacen eso, esa es la tarea de él. Él me ayuda a colgarlas todas, me ayuda a recogerlas, a veces él las quita él solito, él tiene bastante facilidad para ayudar en casa, le gusta organizar su cuarto, le gusta tener su cuarto arreglado, su cama bien arreglada, sus ositos todos encima de la cama, todo arregladito.

I: ¿Él va a comprar? ¿'maneja dinero, conoce los billetes?

M: Él va al mercado, lo conoce, conoce el billete de cien reales, cincuenta, veinte y cinco, eso él lo conoce.

I: ¿Maneja el cambio?

M: Sí, lo maneja, sabe bien todo eso. Puedo darle cincuenta reales y decirle: Hijo, vaya a comprar jugo para mamá, él va y cuando llega, llega con el cambio correcto. Él sabe hacer cuentas, le gusta la matemática.

I: ¿Él ahorra? ¿maneja monedas?

M: Sí, guarda su dinero, toda mesada él la guarda.

I: ¿'Tiene mesada?

M: Cada mes les doy diez reales. Lo guarda.

I: ¿No le gusta andar gastándolo pues?

M: No. Viene él y me dice: "mamá si necesitas dinero, yo te lo presto y tu me lo pagas 
el otro mes". Él me presta dinero y después cuando yo recibo hacemos cuentas con él. No le gusta gastar mucho el dinero. Le gusta gastar dinero comprando carritos, le gustan los carritos. Él hasta tuvo una colección de los Hot Whells, y yo le dije, ¡no tengo dinero para darte tanto!, pero su madrina se los dio como regalo de navidad. (...)

I: ¿¿́l conoce el horario?, ¿la hora? ¿se sitúa en el tiempo?

M: Sí, él lo sabe.

I: Ok.

M: Él se levanta de mañana, por ejemplo, y dice: "mamá, ya es hora de ir para la escuela, son las seis de la mañana”, se levanta, se arregla y va para la escuela. Él sabe más o menos los horarios en que comienzan las caricaturas de la tarde, las novelas que le gustan en la tarde. Él generalmente sabe esas cosas, sabe todo.

Más allá de la gran participación en la vida cotidiana de la familia, mostrándose colaborador y alegre, Rochinha presenta buen conocimiento de los valores monetarios, puede hacer operaciones con billetes y monedas, sin que las operaciones tengan que ser expresadas en forma de registros gráficos, lo que es excesivamente valorizado por la escuela, despreciando en la evaluación de las capacidades matemáticas de los niños sus habilidades de cálculos mentales, aproximaciones, redondeo, estimativas, esa capacidad es revelada en la actividad lúdica realizada en el Centro de Actividades con los juegos como la vendimia, pero sin actividad de registros matemáticos, una vez que las operaciones eran solamente mentales así como apoyadas en la manipulación de los billetes, por medio de composiciones de valores monetarios.

Rochinha, según las palabras de María, tiene un poder de actuar sobre su micromundo a partir de conceptos y procedimientos matemáticos no reconocidos y, por tanto, desvalorizados por la escuela, una escuela que valoriza solamente los conceptos matemáticos que parecen como producciones escritas, justamente en contextos de producciones en que Rochinha tiene dificultad en función de su desarrollo de espacialidad, trazo de su deficiencia auditiva. El problema mayor nos parece ser el hecho de que la escuela reprueba al niño en segundo grado, evaluando solamente sus dificultades y negándose a reconocer todo un potencial para el aprendizaje matemático, lo que constituye la base para el aprendizaje de la misma. Y María concluye "EL SABE TODO", pero un todo no reconocido por la escuela.

Al final, María sintetiza la fase actual de Rochinha, superada la crisis de una vivencia poco productiva en la vida escolar del año anterior:

I: $Y$ hoy, ¿le está gustando la escuela?

M: Hoy sí, gracias a Dios.

I: ¿Por qué cree que hoy le está gustando la escuela? ¿Qué diferencias nota?

M: Porque la profesora le da más atención. 


\section{I: Atención.}

M: Más cariño, ella es más cuidadosa, ella se mantiene en el aula y es muy raro que salga, ella siempre está explicándole, cuando tiene una dificultad ella está allí al lado. La profesora de este año es maravillosa, espero que no la cambien.

I: ¿YY hoy está haciendo las tareas?

M: Sí lo está haciendo solito, ayer incluso hizo toda la tarea solito.

I: ¿Y usted lo apoya?

M: Sí, lo apoyo, él termina de hacer y voy a ver como es que está la tarea, lo que ha hecho. I: ¿Y tiene algún rato en que él se muestra perezoso?

M: A veces dice: “¡Ay, mi mano me duele!” y yo le digo: hijo, debe terminar, después que usted termine puede jugar. Entonces va él y lo termina y vuelve a sus juegos de siempre.

I: Genial. ¿Qué más va a decir de su príncipe?

$\mathrm{M}: \mathrm{Ah}$, mi principito es muy amado, es maravilloso, ahora es niño así, medio con la hermana, pero yo creo que es una fase, ¿verdad? Pero aparte de eso, es un niño dulce, amable y bien dedicado.

Participó de las actividades en el Centro la hermana de Rochinha, Tiana, de 11 años, muy diferente de él, no presentó dificultades significativas, siempre muy involucrada, aplicada y plena de energía y alegría, lo que en cierta forma provoca influencia sobre los comportamientos de Rochinha, pues la mira como una niña de éxito y de plenas capacidades. Pero dejaremos para tratar luego el análisis de las participaciones de Tiana y sus producciones, para concentrarnos a las discusiones posibles sobre su influencia en su hermano. Podemos hipotetizar que sentidos subjetivos producidos en el contexto familiar, articulados con otros en el contexto escolar, tienen participación en la producción de las dificultades de aprendizaje de Rochinha.

\section{Consideraciones}

Hay un avance en este estudio al favorecer la comprensión mas amplia de la constitución del ser matemático como aquel que aprende y produce sentidos subjetivos sobre sus procesos de aprendizaje y de constitución como sujeto inteligente para tratar de desafíos en contextos de resolución de problemas, en especial, de situaciones matemáticas.

Al mirar para esos niños, en el proceso de constitución a lo largo de su vida de construcción social de esos seres humanos, que participaron de las experiencias de matemática lúdica, revela cuánto los esquemas mentales, presentes en una situación matemática dada, no pueden ser comprendidos y explicados, limitándose a la situación en sí, como fenómenos cognitivos puntualmente considerados, una vez que los procesos cognitivos y la dimensión simbólico-emocional de la experiencia encuentran sus fundamentos psicológicos en trayectoria de vida de los sujetos matemáticos, con experiencias y significados que el propio sujeto no tienen conciencia. La búsqueda por la captación de las lógicas de 
constitución subjetiva del ser matemático encuentra en la Epistemología Cualitativa (González Rey, 2002, 2014) herramientas que nos permitieron la producción de informaciones acerca de la génesis de esa compleja constitución del ser matemático como productor de sentidos subjetivos de su capacidad en aprender matemática. La Epistemología Cualitativa favoreció la producción de la información y de la producción teórico-metodológico, exigiendo del investigador un esfuerzo intelectual interpretativo en la búsqueda del reconocimiento de una línea lógica de constitución del sujeto, una línea nada lineal, sino torcida, enredada por las experiencias y sus significados y sentidos por el sujeto en su trayectoria social, colectiva y solidaria.

El estudio permitió la construcción del conocimiento de la constitución subjetiva de esos sujetos, como seres matemáticos, construcción que, infelizmente, educadores y escuelas desconocen, colocando en jaque los cimientos de la definición del diálogo pedagógico, una vez que ese dialogo se revela fragilizado a partir del desconocimiento de quien es ese que aprende, que quiere aprender, en especial, aprender matemática, produciendo sentidos subjetivos, cuyas bases extrapolan sobremanera las relaciones, conocimientos, representaciones, los muros y los tiempos de la escuela.

Eso acaba por revelar como el diálogo en la escuela, como espacio pedagógico o de investigación científica, siempre se muestra limitado y fragilizado, sobre todo en el análisis de las producciones matemáticas de nuestros niños y jóvenes. Esto trae nuevas lógicas y bases epistemológicas para los estudios e investigaciones en Educación Matemática, a partir de las contribuciones de la Teoría de la Subjetividad (González Rey, 2002, 2008, 2012, 2014), haciendo con que pasemos a llevar en consideración, en el complejo proceso de aprendizaje matemático, no solo los sistemas cognitivos de esquemas mentales, sino también la importancia en la definición de los procesos de aprendizaje los sistemas simbólicos-emocionales, que determinan la naturaleza de la producción del conocimiento matemático, calificándolo.

La relación dialógica, constituida por esquema mental-configuración subjetiva, apunta que, si de una parte hay desarrollo de conceptos-procedimientos en el aprendizaje matemático, como vimos en el caso analizado, de otra la relación dialógica genera sentidos subjetivos sobre su capacidad de aprender matemática en el contexto de superación de desafíos y dificultades. El estudio revela que los procesos de pensar y hacer matemática, en especial en la infancia, dependen fuertemente de los sentidos subjetivos del sujeto, sea del sistema simbólico-emocional construido en experiencias pretéritas, o en las experiencias presentes. La visión de si el sujeto posee, la autorrepresentación como ser matemático, impregnado por el complejo sistema simbólico-emocional en la producción de sentidos objetivos, define para el sujeto lo que él puede o debe realizar como matemático, dentro de la escuela y fuera de ella (como fue el caso de este estudio). Sin embargo, fue en la articulación de la Teoría de la Subjetividad, con la valorización del sujeto que aprende como productor de sentidos subjetivos, enlazada al método de la Epistemología Cualitativa que pudimos construir un espacio dialógico con esos pequeños seres matemáticos que nos permitieron traer para el análisis elementos del complejo 
sistema simbólico-emocional, dándonos una nueva lente epistemológica en la forma de ampliar y profundizar la comprensión de los procesos histórico-social-emocional-cognitivo del permanente proceso de constitución del ser matemático: como el niño se percibe como sujeto inteligente, capaz de dar respuestas y validarlas, de extrapolar limites, descubrir alegrías en la posibilidad de estar siempre aprendiendo matemática.

El estudio de muestra como los niños, como es el caso de Rochinha, niño en situación de riesgo social y deficiente auditivo, revela querer aprender de una creencia en la capacidad ontológicamente existente, que fue un importante elemento de análisis observado en nuestros sujetos, que siempre quisieron dar respuestas, mostrándose capaces, niños que, aun en contexto de "riesgo social", tienen madres que, a pesar de su bajo nivel de escolaridad, buscan estimular a sus niños para el aprendizaje y desarrollo pleno. Ese es uno de los motivos por los cuales esos niños se hacen presentes, en el Centro de Actividades, especialmente entusiastas de los talleres de matemática (que siempre las desafían), aun después de la conclusión de la investigación.

Más allá de esos resultados de las relaciones dialógicas y complejas entre producción de sentidos subjetivos y la generación de esquemas mentales, reconocemos, en la constitución de los talleres de matemática lúdica, en el contexto de inclusión social, la oportunidad de los niños concebir la posibilidad de "aprender jugando" en el contexto de un aprendizaje muy distinto de sus experiencias matemáticas escolares. El descubrimiento de esa nueva dimensión de producción y aprendizaje matemática permite un cambio en el propio sistema de producción de sentidos subjetivos de los sujetos. Sus capacidades de aprender matemática y el significado de esos aprendizajes en sus vidas hacen con que el sujeto se enfrente con sistemas de sentidos de subjetividad social de la matemática, llevándola a descubrir que el aprendizaje matemático pude no ser excluyente y más aún, puede ser lúdico.

En ese contexto, la construcción de diálogo con niños fuera de los talleres lúdicos y con sus madres, luego de los análisis microgenéticos de sus registros matemáticos, se mostró de gran valor en el sentido de la ampliación epistemológica de la constitución compleja y tortuosa de esos sujetos que aprenden, algunas veces, aprenden a pesar de la escuela.

Uno de los desafíos que queda abierto al final de este estudio y que nos motiva a la continuidad en el campo de la investigación matemática es traer los estudios de la Teoría de la Subjetividad para la Educación Matemática, en la búsqueda de la comprensión del ser matemático como productor de sentidos subjetivos y de creatividad matemática. Algunas nuevas preguntas nos son impuestas por el estudio realizado:

¿Cuáles son los impactos para la construcción de la praxis pedagógica en matemática, a partir del momento en que el profesor tenga acceso al proceso de constitución del sujeto que aprende con su producción de sentidos subjetivos? 
¿Cuáles son efectivamente los límites y las posibilidades de producción creativa de la matemática llevando en consideración el desarrollo conceptual y de la representación de los conocimientos matemáticos? O sea, ¿cómo la adquisición del lenguaje matemático participa de los procesos creativos en la matemática de Educación Básica?

En fin, este estudio constituyó un desafío de un involucramiento inicial en la Teoría de la Subjetividad como posibilidad de ampliar la comprensión de cada niño como ser matemático como productor de sentidos subjetivos, en un sistema simbólico-emocional que extrapola en mucho las relaciones con la escuela y con el profesor, pero cuya revelación mínima se muestra fundamental para la cualificación de la praxis pedagógica vista como la promoción del aprendizaje matemático.

\section{Referencias.}

Muniz, C. A. (2001). Educação e Linguagem Matemática. In S. M. Bortoni-Ricardo (1ª Ed.), Organização do Trabalho Pedagógico. Brasília: Universidade de Brasília, 1-2, 07-94.

Muniz, C. A. (2009). A produção de notações matemáticas e seu significado. In M. H. Fávero, \& C. Cunha.

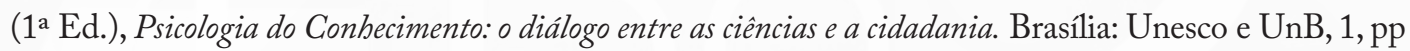
115-143.

Muniz, C. A., \& Bittar, M. (2009). O conceito de "esquema" para um novo olhar para a produção matemática na escola: as contribuiçóes da Teoria dos Campos Conceituais. In C. A. Muniz, \& M. Bitttar (org.), A aprendizagem matemática na perspectiva da Teoria dos Campos Conceituais. 1ed.Curitiba: Editora CRV, 1, 1-93.

Muniz, C. A. (2004). Mediação do conhecimento matemático: re-educação matemática. Revista Ícone, 10, 1, 59-72. ISSN 0104-8104.

Rey, F. G. (2002). Pesquisa Qualitativa em Psicologias: caminhos e desafios. Tradução: Marciel Aristides Ferrara Silva. São Paulo: Pioneira Thomson Learning.

Rey, F. G. (2008). O sujeito que aprende: desafios do desenvolvimento do tema da aprendizagem na psicologia e na prática pedagógica. In M. C. V. R. Tacca (2a Ed.), Aprendizagem e Trabalho Pedagógico. Campinas: Editora Alinea.

Rey, F. G. (2012). A configuração subjetiva dos processos psíquicos: avançando na compreensão da aprendizagem como produção subjetiva. In M. A. Mitjáns, B. J. L. Scoz, \& M. I. S. Castanho, Ensino e Aprendizagem: a subjetividade em foco. Brasília: Liber livros. 
Rey, F. G. (2014). Ideias e modelos teóricos na pesquisa construtivo-interpretativa. In A. M. Mitjáns, \& M. M. V. Neubern (org.), Subjetividade Contemporânea: discussöes epistemológicas e metodológicas. Campinas: Alinea. ISBN 978-857516-703-8

Vergnaud, G. (1994). L'enfant, la mathématique et la réalité. Paris: Peter Lang.

Vergnaud, G. (1998). Quiest-ce que la pensée? dans les actes du Colloque: Quiest-ce que la pensée? Suresne, Laboratoire De Psychologie Cognitive et Activités Finalisées, Université Paris VIII, 1-21.

Vergnaud, G. (2009). O que é aprender? In C. A. Muniz, \& M. Bitttar (1ª Ed.), A aprendizagem matemática na perspectiva da Teoria dos Campos Conceituais. Curitiba: Editora CRV, 1, 1-93. 